\title{
Consultant based Services
}

\author{
One man's experience
}

Alasdair J. Macdonald, Consultant Psychiatrist/Psychotherapist, Crichton Royal Hospital, Dumfries

Current government policy and the need for higher standards of training are leading to reductions in registrar posts and more emphasis on 'consultant based' services. My first consultant post was in such a service. I hope that a description of some of the difficulties encountered will assist informed debate.

In $1980 \mathrm{I}$ was appointed as a second consultant to a seaside town with a population of 100,000 , one-fourth of whom were over 65 years of age. There were many seasonal visitors. Our catchment area was 35 miles from our hospital and our remit was to develop community care from a day hospital base with out-patient facilities. The mentally handicapped and patients under 16 years of age were treated by other teams.

The in-patient service was based on a large rural mental hospital in the next county. It was shared by two consultants from other sectors and cared for a total population of 250,000 . My colleague and I shared responsibility for 24 acute beds, six long-stay wards and our share of multi-user units such as intensive care and rehabilitation. I also supervised an assessment ward for the elderly. All four consultants shared on-call duties for the hospital.

The long-term wards received immediate medical supervision from clinical assistants. Initially our acute beds were covered by the duty doctor in our absence but this was insufficient because so much of our time was spent away from the hospital. We then arranged cover for the acute ward through specified clinical assistant sessions until the apppointment of vocational trainees two years later. The clinical assistants were drawn from a wide variety of backgrounds and training. They were accustomed to act as independent practitioners and the issue of who was ultimately responsible for the patient's care was never fully resolved. Although the practice is officially unacceptable the hospital's night cover was provided by nocturnal clinical assistant sessions. The cover this provided was minimal and detracted from the sessions available for day time patient care.

Outside the hospital all emergencies out of hours were attended by the consultants, being the only staff with 24-hour responsibility. Such calls were initially very frequent. County Social Services sought a consultant opinion prior to every application under the Mental Health Act. This gave rise to many night calls which were often timeconsuming, the more so because the social worker on call was based $\mathbf{4 0}$ miles away. In effect, until trainees were appointed, we worked not as consultant psychiatrists but as general practitioners with an interest in psychiatry.
Because our in-patient facilities were under a different Health Authority our administrative load was doubled. Since community care was a new venture for this area, planning future developments in consultation with Health Service and local authority managers formed a major part of our activities. There was disagreement between the two Authorities concerned about financial responsibility for our service which resulted in many uncertainties affecting forward planning.

An additional burden arose when the declining holiday trade led to an increase in long-term accommodation. Many patients from our rehabilitation unit were discharged into local accommodation but were not followed up by their own sector teams who knew them best. As private and local authority residential provision increased many agencies outside the area made use of these places without making any arrangements for follow-up care.

Thanks to the good offices of our general hospital colleagues and the local University Department of Mental Health we obtained three vocational training senior house officer (SHO) posts, one attached to our sector. Care within the hospital improved greatly at the cost of additional teaching. Being prospective general practitioners, our SHOs were eager for community experience and we encouraged supervised out-patient and day hospital work. However, we would not ask them to undertake crisis calls in the community without supervision and such supervision increased our workload rather than reducing it. We found general practitioners unwilling to accept psychiatric opinions from vocational trainees unless the consultant had also seen the patient.

Vocational trainees differ in their motivations from career SHO/registrars. However enthusiastic, our trainees could not learn enough in six months to work in specialised areas such as intensive care wards or rehabilitation units which had to remain the responsibility of clinical assistants. The SHOs were not skilled enough to be responsible for psychological treatments such as group therapy or family therapy. As a result, in the Day Hospital, many psychological treatments were carried out by non-medical staff with a minimum of supervision from ourselves. Being a new service, there was no existing philosophy for the unit to which staff could relate and for the most part our staff were responsible to managers with little or no experience of psychiatry. The result was a high quality of care at the cost of considerable anxiety for our staff (and ourselves). Staff support was therefore another major role for the consultants. 
The limitations of our in-patient service resulted in a shift towards crisis intervention and home care, especially for the elderly population. This increased our already substantial commitment to domiciliary visits. It was rare for a day to pass without one or more urgent referrals. The clinical assistants in the Day Hospital were heavily committed within that unit and could not offer emergency cover. Community psychiatric nurses and our own social workers proved the most versatile staff and could often contain emergencies if no psychiatrist was available.

Even with SHOs, therefore, each consultant was acting as his own registrar. This meant not only doing different tasks but actually doing the work of two doctors for much of the time. Unlike clinical assistants, registrars can work flexible hours and provide night cover. Experienced registrars have the skills necessary to carry out psychological treatments, to provide emergency opinions and to teach and support other staff. Clinical responsibility and responsibility for supervision remain with the consultant who thus also remains informed about the total service. Where non-consultant staff function independently of the consultants, continuity of care and team work diminish.

Without registrars, consultant cover becomes a major issue. During any absence, such as annual leave, one consultant can cover but not replace another because of differences in clinical practice. The standard of care falls then to what an SHO can provide. Similarly, no one can replace the consultant in speaking for his own unit and speciality in his administrative role. Senior professionals from other disciplines will not delay their deliberations to wait for the psychiatrist who has been called to a clinical emergency. Yet someone with sufficient skill must assess the patient until the consultant's opinion can be obtained. In our case attendance at local meetings was made possible by radiopaging devices. Longer absences placed an intolerable burden on one's colleagues. I sought employment elsewhere when it became apparent that there was no prospect of improvement in this situation.

It may be argued that our staffing was insufficient and that more medical staff would have reduced these problems.
But all plans for consultant-based services so far proposed show an absolute reduction in available medical staff time. Furthermore, clinical assistants are limited to certain hours. Associate specialist posts are daily fewer in number and are unpopular politically. Additional 'junior' consultants to do the work of registrars is a very expensive solution. Appointing more consultants, each with a smaller catchment area, means that facilities such as day hospitals must be shared, with corresponding problems for other staff and for continuity of care. Cover remains a problem and this solution is both costly and politically unrealistic.

To abandon sectorisation and catchment areas, as some have suggested, would be a partial solution. The general practitioner would then have alternatives if a particular consultant was unavailable. Expediency and the consultant's style of practice would govern referrals. However, all the local knowledge gained from sectorisation and close teamwork would be lost and equitable resource allocation would become more difficult. In the long run patient care would suffer.

My conclusion is that any change from the present system of consultant and registrar means either that fewer patients are treated or that the same number are treated but less effectively. This represents a shift from a demand-based service to a supply-based service. This is a major philosophical shift for the National Health Service but is in agreement with the effects of current government policy. The message is not 'treat the sick' but 'do what you can'. This is a pragmatic approach which appeals to me personally but is hardly the NHS as originally conceived. It is the voice of the Third World, not the democratic utopia of Aneurin Bevan.

For economic reasons the change may be unavoidable, but no one should assume that calling a service "consultant based' will by magic make it equivalent to the present service based around consultants. Because of its relative impoverishment over generations by comparison with general medical specialists, psychiatry will fare worst under such a system.

\section{(See page 68)}

\section{'Doctors and Patients'}

This is a series of six programmes from Central being screened on the ITV network on Mondays at 12.30 p.m. from 23 March 1987. The programmes feature six different medical teams who combine a variety of treatments so that caring for the whole person becomes an inseparable part of curing the illness. A cancer ward, a pain management course, a child development unit, an inner-city hospital, a general practice and a therapeutic centre using complemen- tary medicine are included. 'Doctors and Patients' asks how necessary is this wholistic form of treatment and whether it is the direction in which orthodox medicine is heading in the future. A booklet will be published to coincide with the series and is obtainable from 'Doctors and Patients', PO Box 96, Birmingham B1 $2 \mathrm{JL}$ on receipt of a stamped addressed envelope. 